\title{
GC-MS Analysis of Bio-active Compounds in Ethanol Extract of Putranjiva roxburghii Wall. Fruit Peel
}

\author{
Md. Mahmudul Hasan*, Md. Rezuan Al Mahmud, Md. Gaziul Islam
}

Md. Mahmudul Hasan*, Md. Rezuan Al Mahmud, Md. Gaziul Islam

Molecular Biology and Protein Science Laboratory, Department of Genetic Engineering and Biotechnology, Faculty of Life and Earth Sciences, University of Rajshahi, Rajshahi-6205, BANGLADESH.

Correspondence

Mr. Md. Mahmudul Hasan

Molecular Biology and Protein Science Laboratory, Department of Genetic Engineering and Biotechnology, Faculty of Life and Earth Sciences, University of Rajshahi, Rajshahi-6205, BANGLADESH.

Phone no : +8801720214658

E-mail: mahmudul_geb@yahoo.com

History

- Submission Date: 28-08-2018;

- Review completed: 15-10-2018,

- Accepted Date: 06-11-2018

DOI : 10.5530/pj.2019.1.24

Article Available online

http://www.phcogj.com/v11/i1

\section{Copyright}

(C) 2019 Phcog.Net. This is an openaccess article distributed under the terms of the Creative Commons Attribution 4.0 International license.

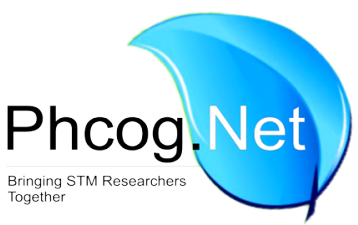

\section{ABSTRACT}

Introduction: Putranjiva roxburghii Wall. has long been used in folkloric medicine for treating arthralgia, fever, hemorrhoids, muscle pain and rheumatism. But no reports were found regarding phytochemical constituents in P. roxburghii fruit peel. Therefore, this study was designed to analyze extract of $P$. roxburghii fruit peel (PRFP). Methods: Gas chromatography-mass spectrometry (GC-MS) analysis of the ethanol extract of PRFP was carried out by using a GC-MS equipment. Results: The GC-MS analysis has revealed the existence of different phytochemical compounds in the ethanolic extract of PRFP. The major compounds in PRFP extract are Cyclohexanol, 5-methyl-2-(1-methylethenyl)- (4.56\%), 6-Octen-1-ol, 3,7-dimethyl- (41.07\%), Geraniol (2.45\%), (1R,2S,5R)-2-(2-Hydroxy-2-propanyl)-5-methylcyclohexanol (14.09\%), 2,6-Octadiene, 2,6-dimethyl- (7.04\%), p-Menthane-3,8-diol, cis-1,3, trans-1,4- (3.39\%), 2,6-Octadien-1-ol, 3,7-dimethyl-, acetate (6.69\%) and 13-Docosenamide, (Z)- $(2.83 \%)$. A total of 25 compounds identified representing $99.98 \%$ of total ethanolic extract. Conclusion: Overall finding suggests that PRFP contain various phytocomponents and is recommended as an important source of pharmaceutical ingredients.

Key words: Putranjiva roxburghii, Fruit peel, Ethanol extract, GC-MS, Pharmaceutical ingredients.

\section{INTRODUCTION}

Putranjiva roxburghii Wall. belongs to the Euphorbiaceae family. It is widely grown in Bangladesh, India, Indochina, Myanmar, Nepal, Sri Lanka and Thailand. ${ }^{1}$ It is locally known as "Putranjiv" in Bangladesh. This is an ornamental plant of tropical India and known as child life tree. ${ }^{2}$ The seed of this plant is a good source of well stable and potent trypsin inhibitor. ${ }^{2}$ The seed is also a potent source of thermostable glycosyl hydrolase family 1 enzyme with $\beta$-D-glucosidase and $\beta$-D-galactosidase activities. ${ }^{3}$ Spreading the leaves of this plant over the floor of maternity room has been reported for an easy delivery. ${ }^{4}$ In Thailand, P. roxburghii fruits and leaves have long been traditionally used for the treatment of arthralgia, fever, muscle pain and rheumatism and the entire plant has also been used for the treatment of hemorrhoids and fever. ${ }^{1,5} P$. roxburghii leaf has analgesic, anti-inflammatory and antipyretic activities. ${ }^{6}$ Though $P$. roxburghii has long been used in folkloric treatment, no studies have been conducted regarding the presence of phytocompounds in $P$. roxburghii fruit peel. Thereafter, this current study was aimed to investigate the bioactive phytocompounds in the fruit peel extract of $P$. roxburghii.

\section{MATERIALS AND METHODS}

\section{Chemicals and reagents}

All of the chemicals and reagents used in this study were of analytical grade.

\section{Collection of plant material}

The fruit of Putranjiva roxburghii plant was collected from the University of Rajshahi (Rajshahi, Bangladesh) campus in October 2017. Then the fruit was identified and authenticated by a taxonomist Mr. Md. Arshed Alam (Department of Botany, University of Rajshahi, Rajshahi, Bangladesh). A herbarium of the P. roxburghii containing fruit was prepared and preserved in the Herbarium of the Department of Botany, University of Rajshahi, Rajshahi, Bangladesh under an accession number 00312 for further referencing.

\section{Preparation of extract}

The peel was separated from the fruits and was dried by using a drying cabinet (LJ-120A(S), Guangdong LIK Industry Co., Ltd., China) at $37^{\circ} \mathrm{C}$ temperature. The dried peel was grinded to make fine powder. Then extraction was performed by dissolving the powder $(5 \mathrm{~g})$ in $50 \mathrm{ml}$ of $95 \%$ ethanol. The content was sonicated by using sonicator (Soniprep 150, China) at $20 \mathrm{kHz}$ for $10 \mathrm{~min}$. Extract was filtered using Glass Fiber Filter paper (Macherey NAGEL, GmBH, German) with DURAN ${ }^{\circ}$ Filtering Apparatus (German) at room temperature. The resulting solution was concentrated with VirTis BenchTop Pro Freeze Dryer (German). Finally, the extract was stored in a refrigerator at $4^{\circ} \mathrm{C}$ for further use.
Cite this article: Hasan MM, Mahmud MRA, Islam MG. GC-MS Analysis of Bio-active Compounds in Ethanol Extract of Putranjiva roxburghii Wall. Fruit Peel. Pharmacog J. 2019;11(1):146-9. 


\section{GC-MS analysis}

The phytochemical investigation of ethanolic extract was carried out on a GC-MS equipment (GCMS-QP2020; SHIMADZU) comprising an AOC-20s auto-sampler, an AOC-20i auto-injector and a Gas Chromatograph (GC-2010 Plus) interfaced to a Mass Spectrometer. Experimental conditions of GC-MS system were as follows: SH-Rxi-5Sil-MS capillary standard non-polar column, dimension: $30 \mathrm{~m}$, ID: $0.25 \mathrm{~mm}$, film thickness: $0.25 \mu \mathrm{m}$. Flow rate of mobile phase (carrier gas: $\mathrm{He}$ ) was set at $1.7 \mathrm{ml} / \mathrm{min}$. In the gas chromatography part, temperature profile (initial temperature) was $80^{\circ} \mathrm{C}$ (hold time $2.0 \mathrm{~min}$ ) rose to $150^{\circ} \mathrm{C}$ at $5^{\circ} \mathrm{C} / \mathrm{min}$ (hold time $5.0 \mathrm{~min}$ ) and finally the temperature was increased to $280^{\circ} \mathrm{C}$ at $5^{\circ} \mathrm{C}$ (hold time $8.0 \mathrm{~min}$ ). The injection volume was $5 \mu \mathrm{l}$. Sample dissolved in methanol was run at a range of $45-350 \mathrm{~m} / \mathrm{z}$ and the results were compared by using National Institute of Standards and Technology such as NIST08, NIST08s and NIST14 Library Search Programmes.

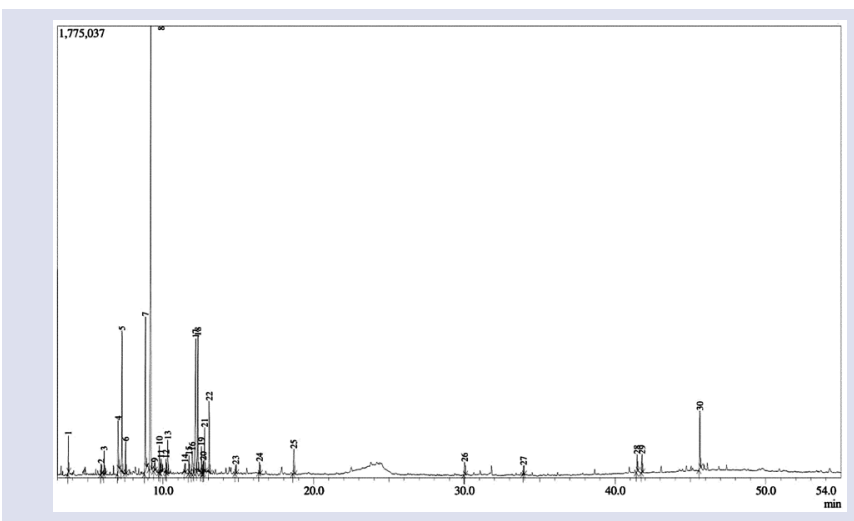

Figure 1: GC-MS chromatogram of PRFP ethanol extract.

Table 1: List of compounds in PRFP ethanol extract identified by using GC-MS.

\begin{tabular}{|c|c|c|c|c|c|c|}
\hline $\begin{array}{c}\text { Com. } \\
\text { no. }\end{array}$ & Peak no. & Name of compounds & $\begin{array}{l}\text { Ret. } \\
\text { time } \\
\text { (min) }\end{array}$ & $\begin{array}{l}\text { Molecular } \\
\text { formula }\end{array}$ & $\begin{array}{l}\text { Molecular } \\
\text { weight } \\
\text { (g/mol) }\end{array}$ & $\begin{array}{c}\text { Peak } \\
\text { Area (\%) }\end{array}$ \\
\hline 1 & 1 & 3,4-Hexanediol, 2,5-dimethyl- & 3.714 & $\mathrm{C}_{8} \mathrm{H}_{18} \mathrm{O}_{2}$ & 146.227 & 1.53 \\
\hline \multirow[t]{2}{*}{2} & 2 & Linalool & 5.890 & $\mathrm{C}_{10} \mathrm{H}_{18} \mathrm{O}$ & 154.253 & 0.45 \\
\hline & 3 & Linalool & 6.094 & $\mathrm{C}_{10} \mathrm{H}_{18} \mathrm{O}$ & 154.253 & 0.89 \\
\hline 3 & 4 & Citronellal & 7.016 & $\mathrm{C}_{10} \mathrm{H}_{18} \mathrm{O}$ & 154.253 & 1.86 \\
\hline \multirow[t]{2}{*}{4} & 5 & Cyclohexanol, 5-methyl-2-(1-methylethenyl)- & 7.275 & $\mathrm{C}_{10} \mathrm{H}_{18} \mathrm{O}$ & 154.253 & 3.97 \\
\hline & 6 & Cyclohexanol, 5-methyl-2-(1-methylethenyl)- & 7.514 & $\mathrm{C}_{10} \mathrm{H}_{18} \mathrm{O}$ & 154.253 & 0.59 \\
\hline \multirow[t]{2}{*}{5} & 7 & 6-Octen-1-ol, 3,7-dimethyl- & 8.829 & $\mathrm{C}_{10} \mathrm{H}_{20} \mathrm{O}$ & 156.265 & 8.53 \\
\hline & 8 & 6-Octen-1-ol, 3,7-dimethyl- & 9.192 & $\mathrm{C}_{10} \mathrm{H}_{20} \mathrm{O}$ & 156.265 & 32.54 \\
\hline 6 & 9 & 2,6-Octadienal, 3,7-dimethyl-, (Z)- & 9.445 & $\mathrm{C}_{10} \mathrm{H}_{16} \mathrm{O}$ & 152.237 & 0.43 \\
\hline 7 & 10 & Geraniol & 9.756 & $\mathrm{C}_{10} \mathrm{H}_{18} \mathrm{O}$ & 154.253 & 2.45 \\
\hline 8 & 11 & 4-Isopropyl-1,3-cyclohexanedione & 9.851 & $\mathrm{C}_{9} \mathrm{H}_{14} \mathrm{O}_{2}$ & 154.209 & 0.50 \\
\hline 9 & 12 & 2,6-Octadienal, 3,7-dimethyl-, (E)- & 10.192 & $\mathrm{C}_{10} \mathrm{H}_{16} \mathrm{O}$ & 152.237 & 1.19 \\
\hline 10 & 13 & 6-Octen-1-ol, 3,7-dimethyl-, formate & 10.319 & $\mathrm{C}_{11} \mathrm{H}_{20} \mathrm{O}_{2}$ & 184.279 & 1.65 \\
\hline 11 & 14 & (R)-(+)-Citronellic acid & 11.453 & $\mathrm{C}_{10} \mathrm{H}_{18} \mathrm{O}_{2}$ & 170.252 & 0.70 \\
\hline 12 & 15 & (1R,2S,5R)-2-(2-Hydroxy-2-propanyl)-5-methylcyclohexanol & 11.723 & $\mathrm{C}_{10} \mathrm{H}_{20} \mathrm{O}_{2}$ & 172.265 & 1.17 \\
\hline 13 & 16 & 2,6-Octadiene, 2,6-dimethyl- & 11.933 & $\mathrm{C}_{10} \mathrm{H}_{18}$ & 138.254 & 1.46 \\
\hline 12 & 17 & (1R,2S,5R)-2-(2-Hydroxy-2-propanyl)-5-methylcyclohexanol & 12.169 & $\mathrm{C}_{10} \mathrm{H}_{20} \mathrm{O}_{2}$ & 172.265 & 12.92 \\
\hline 13 & 18 & 2,6-Octadiene, 2,6-dimethyl- & 12.317 & $\mathrm{C}_{10} \mathrm{H}_{18}$ & 138.254 & 5.58 \\
\hline 14 & 19 & 2,6-Octadien-1-ol, 3,7-dimethyl-, acetate, (Z)- & 12.549 & $\mathrm{C}_{12} \mathrm{H}_{20} \mathrm{O}_{2}$ & 196.290 & 1.90 \\
\hline 15 & 20 & Geranyl acetate & 12.670 & $\mathrm{C}_{12} \mathrm{H}_{20} \mathrm{O}_{2}$ & 196.290 & 1.33 \\
\hline 16 & 21 & p-Menthane-3,8-diol, cis-1,3,trans-1,4- & 12.759 & $\mathrm{C}_{10} \mathrm{H}_{20} \mathrm{O}_{2}$ & 172.268 & 3.39 \\
\hline 17 & 22 & 2,6-Octadien-1-ol, 3,7-dimethyl-, acetate & 13.062 & $\mathrm{C}_{12} \mathrm{H}_{20} \mathrm{O}_{2}$ & 196.290 & 6.69 \\
\hline 18 & 23 & 3-Hydroxy-4-methoxybenzyl alcohol & 14.820 & $\mathrm{C}_{8} \mathrm{H}_{10} \mathrm{O}_{3}$ & 154.165 & 0.38 \\
\hline 19 & 24 & $\beta$-Bisabolene & 16.400 & $\mathrm{C}_{15} \mathrm{H}_{24}$ & 204.357 & 0.49 \\
\hline 20 & 25 & Caryophyllene oxide & 18.685 & $\mathrm{C}_{15} \mathrm{H}_{24} \mathrm{O}$ & 220.356 & 0.90 \\
\hline 21 & 26 & Hexadecanoic acid, methyl ester & 30.005 & $\mathrm{C}_{17} \mathrm{H}_{34} \mathrm{O}_{2}$ & 270.457 & 0.79 \\
\hline 22 & 27 & 11-Octadecenoic acid, methyl ester & 33.929 & $\mathrm{C}_{19} \mathrm{H}_{36} \mathrm{O}_{2}$ & 296.495 & 0.33 \\
\hline 23 & 28 & Hexadecanoic acid, 2-hydroxy-1-(hydroxymethyl) ethyl ester & 41.466 & $\mathrm{C}_{19} \mathrm{H}_{38} \mathrm{O}_{4}$ & 330.509 & 0.66 \\
\hline 24 & 29 & Bis(2-ethylhexyl) phthalate & 41.790 & $\mathrm{C}_{24} \mathrm{H}_{38} \mathrm{O}_{4}$ & 390.564 & 1.88 \\
\hline 25 & 30 & 13-Docosenamide, $(\mathrm{Z})$ - & 45.631 & $\mathrm{C}_{22} \mathrm{H}_{43} \mathrm{NO}$ & 337.592 & 2.83 \\
\hline
\end{tabular}


Table 2: Bio-activity of compounds identified in PRFP ethanol extract by GC-MS.

\begin{tabular}{|c|c|c|}
\hline Com. no. & Name of compounds & Bio-activity \\
\hline 1 & 3,4-Hexanediol, 2,5-dimethyl- & Not reported \\
\hline 2 & Linalool & $\begin{array}{l}\text { Anticancer, antimicrobial, anti-inflammatory, anti-hyperlipidemic, } \\
\text { antinoceptive, analgesic, anxiolytic, antidepressive and } \\
\text { neuroprotective }{ }^{7}\end{array}$ \\
\hline 3 & Citronellal & $\begin{array}{l}\text { Antioxidant, antimicrobial, anthelmintic, anticonvulsant } \\
\text { antitrypanosomal, wound healing and mosquito repellent }{ }^{8}\end{array}$ \\
\hline 4 & Cyclohexanol, 5-methyl-2-(1-methylethenyl)- & $\begin{array}{c}\text { Antimicrobial, anticancer, anti-tumor, analgesic, anti-inflammatory, } \\
\text { sedative, antifungal, hypocholesterolemic, insecticide, insectifuge } \\
\text { chemo preventive and pesticidal }{ }^{9}\end{array}$ \\
\hline 5 & 6-Octen-1-ol, 3,7-dimethyl- & $\begin{array}{l}\text { Antinociceptive, anti-inflammatory, antifungal, repellent and } \\
\qquad \text { larvicidal }^{10}\end{array}$ \\
\hline 6 & 2,6-Octadienal, 3,7-dimethyl-, (Z)- & Antimicrobial $^{11}$ \\
\hline 7 & Geraniol & $\begin{array}{l}\text { Anticancer, antibacterial, anthelmintic, anti-inflammatory and } \\
\text { anticarcinogenic }\end{array}$ \\
\hline 8 & 4-Isopropyl-1,3-cyclohexanedione & Not reported \\
\hline 9 & 2,6-Octadienal, 3,7-dimethyl-, (E)- & Antimicrobial $^{11}$ \\
\hline 10 & 6-Octen-1-ol, 3,7-dimethyl-, formate & Antitumor, antibacterial, antifungal, flavoring and fragrance ${ }^{13-16}$ \\
\hline 11 & (R)-(+)-Citronellic acid & Anticancer ${ }^{17}$ \\
\hline 12 & (1R,2S,5R)-2-(2-Hydroxy-2-propanyl)-5-methylcyclohexanol & Anticancer, antimicrobial, anti-inflammatory and flavour enhancing ${ }^{18}$ \\
\hline 13 & 2,6-Octadiene, 2,6-dimethyl- & Not reported \\
\hline 14 & 2,6-Octadien-1-ol, 3,7-dimethyl-, acetate, (Z)- & Anticancer and antinociceptive $e^{19,20}$ \\
\hline 15 & Geranyl acetate & Anticancer and antinociceptive $e^{19,20}$ \\
\hline 16 & p-Menthane-3,8-diol, cis-1,3,trans-1,4- & Mosquito repellent ${ }^{21}$ \\
\hline 17 & 2,6-Octadien-1-ol, 3,7-dimethyl-, acetate & Anticancer ${ }^{20}$ \\
\hline 18 & 3-Hydroxy-4-methoxybenzyl alcohol & Not reported \\
\hline 19 & $\beta$-Bisabolene & Anticancer ${ }^{22}$ \\
\hline 20 & Caryophyllene oxide & $\begin{array}{l}\text { Anticancer, antioxidant, anti-inflammatory immunomodulator, } \\
\text { cytoprotective and trypanocidal }{ }^{23}\end{array}$ \\
\hline 21 & Hexadecanoic acid, methyl ester & Antioxidant, antibacterial and antifungal ${ }^{24,25}$ \\
\hline 22 & 11-Octadecenoic acid, methyl ester & Not reported \\
\hline 23 & Hexadecanoic acid, 2-hydroxy-1-(hydroxymethyl) ethyl ester & Antioxidant, anti-inflammatory, anthelmintic ${ }^{25}$ \\
\hline 24 & Bis(2-ethylhexyl) phthalate & Cytotoxic ${ }^{26}$ \\
\hline 25 & 13-Docosenamide, $(\mathrm{Z})$ - & Antiviral $^{26}$ \\
\hline
\end{tabular}

\section{RESULTS AND DISCUSSION}

The GC-MS Chromatogram revealed a total of 30 peaks with different retention times (Figure 1). Though the peak number is 30, the identified compounds are 25 due to the reiteration of 5 compounds (Table 1). The large compound fragments compared to small compounds gave taller appearance of peaks at different $\mathrm{m} / \mathrm{z}$ ratios.

However, the identified compounds in the extract of PRFP are 3,4Hexanediol,2,5-dimethyl-, Linalool, Citronellal, Cyclohexanol,5-methyl2-(1-methylethenyl)-, 6-Octen-1-ol,3,7-dimethyl-, 2,6-Octadienal,3,7dimethyl-,(Z)-, Geraniol, 4-Isopropyl-1,3-cyclohexanedione, 2,6-Octadienal, 3,7-dimethyl-,(E)-, 6-Octen-1-ol,3,7-dimethyl-, formate, (R)-(+)-Citronellic acid, (1R,2S,5R)-2-(2-Hydroxy-2-propanyl)-5-methylcyclohexanol, 2,6-Octadiene,2,6-dimethyl-, 2,6-Octadien-1-ol,3,7-dimethyl-,acetate,(Z)-, Geranyl acetate, p-Menthane-3,8-diol,cis-1,3,trans-1,4-, 2,6-Octadien-1-ol,3,7-dimethyl-,acetate, 3-Hydroxy-4-methoxybenzyl alcohol, $\beta$-Bisabolene, Caryophyllene oxide, Hexadecanoic acid, methyl ester, 11-Octadecenoic acid, methyl ester, Hexadecanoic acid, 2-hydroxy-1(hydroxymethyl) ethyl ester, Bis(2-ethylhexyl) phthalate and 13-Docosenamide, $(Z)$ - (Table 1). The composition determined for the PRFP extract corresponds to $99.98 \%$ of the whole GC-MS chromatogram. The identified compounds were previously reported with numerous important bio-activities (Table 2).

\section{CONCLUSION}

The identified compounds after GC-MS analysis of P. roxburghii fruit peel extract justifies the use of fruit peel by traditional practitioner. However, isolation of each single compounds and subjecting it to the biological activity will definitely give fruitful outcomes as the identified compounds were previously reported with important bio-activity. Therefore, this plant is recommended as a source of phytopharmaceutical value.

\section{ACKNOWLEDGEMENT}

We are thankful to Mr. Md. Abdul Jalil (Research Chemist, Bangladesh Council of Scientific and Industrial Research, Rajshahi Division, Rajshahi, Bangladesh) for giving us an opportunity to use GC-MS in his laboratory.

\section{CONFLICT OF INTEREST}


The authors declare no conflict of interest.

\section{ABBREVIATIONS}

GC-MS: Gas chromatography-Mass spectrometry; PRFP: P. roxburghii Fruit Peel.

\section{REFERENCES}

1. Phuphathanaphong L. Flor a of Thailand Euphorbiaceae. Natl Herb Nederland. 2006;336:1877-87.

2. Chaudhary NS, Shee C, Islam A, Ahmad F, Yernool D, Kumar P, et al. Purification and characterization of a trypsin inhibitor from Putranjiva roxburghii seeds. Phytochemistry. 2008;69(11):2120-6.

3. Patel GK, Kar B, Sharma AK. Characterization of a thermostable family 1 Glycosyl Hydrolase enzyme from Putranjiva roxburghii seeds. Applied Biochemistry and Biotechnology. 2012;166(3):523-35

4. Singh H, Bisht GS. Some novel folk treatments among the Tribes of Uttar Pradesh. Ancient science of Life. 1999;18(3-4):250-53.

5. Boonyaprapat N, Chokechaicharoenporn A. Samunprai Maipuenban. Prachachon, Bangkok. 1999;3:262-73.

6. Reanmongkol W, Noppapan T, Subhadhirasakul S. Antinociceptive, antipyretic, and anti-inflammatory activities of Putranjiva roxburghii Wall. leaf extract in experimental animals. Journal of Natural Medicines. 2009;63(3):290-9.

7. Pereira I, Severino P, Santos AC, Silva AM, Souto EB. Linalool Bioactive Properties and Potential Applicability in Drug Delivery Systems. Colloids and Surfaces B: Biointerfaces. 2018.

8. Sharma R, Rao R, Kumar S, Mahant S, Khatkar S. Therapeutic potential of Citronella Essential Oil: a review. Current Drug Discovery Technologies. 2018.

9. Rency RC, Vasantha K, Maruthasalam A. Identification of bioactive compounds from ethanolic leaf extracts of Premnaser ratifolia L. using GC-MS. Bioscience Discovery. 2015;6(2):96-101.

10. Pereira FD, Mendes JM, Lima IO, Mota KS, Oliveira WA, Lima ED. Antifungal activity of geraniol and citronellol, two monoterpenes alcohols, against Trichophyton rubrum involves inhibition of ergosterol biosynthesis. Pharmaceutical Biology. 2015;53(2):228-34

11. Dubey D, Patnaik R, Ghosh G, Padhy RN. In vitro Antibacterial Activity, Gas Chromatography-Mass Spectrometry Analysis of Woodfordia fruticosa Kurz. Leaf Extract and Host Toxicity Testing With In vitro Cultured Lymphocytes From Human Umbilical Cord Blood. Osong Public Health and Research Perspectives. 2014;5(5):298-312.

12. Singh S, Fatima Z, Ahmad K, Hameed S. Fungicidal action of geraniol against Candida albicans is potentiated by abrogated $\mathrm{CaCdr} 1 \mathrm{p}$ drug efflux and fluconazole

\section{GRAPHICAL ABSTRACT}

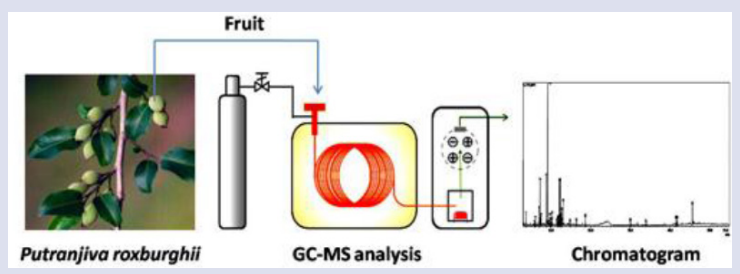

synergism. PloS One. 2018;13(8):e0203079

13. Fang $\mathrm{HJ}$, Su XL, Liu HY, Chen $\mathrm{YH}, \mathrm{Ni} J \mathrm{JH}$. Studies on the chemical components and anti-tumour action of the volatile oils from Pelargonium graveoleus. Yao Xuexuebao = Acta Pharmaceutica Sinica. 1989;24(5):366-71.

14. Rath CC, Dash SK, Rao BR. Antifungal activity of rose-scented geranium (Pelargonium species) essential oil and its six constituents. Journal of Essential Oil Bearing Plants. 2005;8(2):218-22

15. Choi HS. Characteristic odor components of kumquat (Fortunella japonica Swingle) peel oil. Journal of Agricultural and Food Chemistry. 2005;53(5):1642-7.

16. Bigos M, Wasiela M, Kalemba D, Sienkiewicz M. Antimicrobial activity of geranium oil against clinical strains of Staphylococcus aureus. Molecules. 2012;17(9) 10276-91.

17. Rychlicka M, Niezgoda N, Gliszczynska A. Lipase-Catalyzed Acidolysis of Egg-Yolk Phosphatidylcholine with Citronellic Acid. New Insight into Synthesis of Isoprenoid-Phospholipids. Molecules. 2018;23(2):314.

18. Kamatou GP, Vermaak I, Viljoen AM, Lawrence BM. Menthol: a simple monoterpene with remarkable biological properties. Phytochemistry. 2013;96:15-25.

19. Quintans-Júnior L, Moreira JC, Pasquali MA, Rabie S, Pires AS, Schröder R, et al Antinociceptive activity and redox profile of the monoterpenes. ISRN Toxicology. 2013.

20. Qi F, Yan Q, Zheng Z, Liu J, Chen Y, Zhang G. Geraniol and geranyl acetate induce potent anticancer effects in colon cancer Colo-205 cells by inducing apoptosis, DNA damage and cell cycle arrest. Journal of BUON.: Official Journal of the Balkan Union of Oncology. 2018;23(2):346-52

21. Webb CE, Hess IM. A review of recommendations on the safe and effective use of topical mosquito repellents. Public Health Res Pract. 2016;26(5):e2651657.

22. Yeo SK, Ali AY, Hayward OA, Turnham D, JacksonT, Bowen ID, et al. $\beta$-Bisabolene, a Sesquiterpene from the Essential Oil Extract of Opoponax (Commiphora guidottii), Exhibits Cytotoxicity in Breast Cancer Cell Lines. Phytotherapy Research. 2016;30(3):418-25.

23. Moreno ÉM, Leal SM, Stashenko EE, García LT. Induction of programmed cel death in Trypanosoma cruzi by Lippia alba essential oils and their major and synergistic terpenes (citral, limonene and caryophyllene oxide). BMC Complementary and Alternative Medicine. 2018;18(1):225.

24. Chandrasekaran M, Senthilkumar A, Venkatesalu V. Antibacterial and antifunga efficacy of fatty acid methyl esters from the leaves of Sesuviumportula castrum L. European Review for Medical and Pharmacological Sciences. 2011;15(7):775-80.

25. Chen Y, Zhou D, Qi D, Gao Z, Xie J, Luo Y. Growth Promotion and Disease Suppression Ability of a Streptomyces sp. CB-75 from Banana Rhizosphere Soil. Frontiers in Microbiology. 2018;8:2704.

26. Wang Y, Ren W, LiY, XuY, Teng Y, Christie P, et al. Non targeted metabolomic analysis to unravel the impact of di (2-ethylhexyl) phthalate stress on root exudates of alfalfa (Medicago sativa). Science of the Total Environment. 2019;646:212-9.

\section{SUMMARY}

- The bioactive constituents of ethanol extract of Putranjiva roxburghii fruit peel was evaluated using GC-MS. A total of 25 compounds were identified and the most abundant compound is 6-Octen-1-ol, 3,7-dimethyl- (41.07\%). However, fruit peel extract from this plant may become as a good source of new drugs for therapeutic studies.

\section{ABOUT AUTHORS}

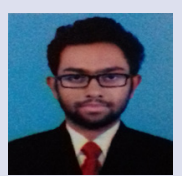

Md. Gaziul Islam: Is an undergraduate research student who has a big desire on Molecular Microbiology.

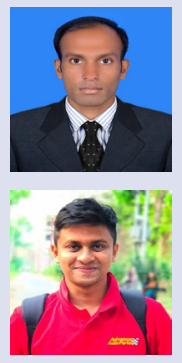

Md. Mahmudul Hasan: Is a research personal who is searching for sources of natural and safe product that may have potent therapeutic effect to combat against cancer. Other areas of his research interest are Molecular Pathology, Redox Regulation, Drug Design and Nutrigenomics.

Md. Rezuan Al Mahmud: Currently working as a Scientific Officer in Globe Biotech Limited (Bangladesh) which focuses on the Drug Design and Development. He has great interest on Molecular Biology and Drug Design.

Cite this article: Hasan MM, Mahmud MRA, Islam MG. GC-MS Analysis of Bio-active Compounds in Ethanol Extract of Putranjiva roxburghii Wall. Fruit Peel. Pharmacog J. 2019;11(1):146-9. 